\title{
Alteration of the Occlusal Vertical Dimension for Prosthetic Restoration Using a Target Tracking System
}

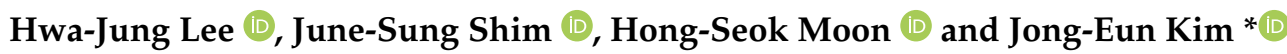 \\ Department of Prosthodontics, Yonsei University College of Dentistry, Yonsei-ro 50-1, Seodaemun-gu, \\ Seoul 03722, Korea; hjlee0227@yuhs.ac (H.-J.L.); jfshim@yuhs.ac (J.-S.S.); hsm5@yuhs.ac (H.-S.M.) \\ * Correspondence: gomyou@yuhs.ac; Tel.: +82-2-2228-3160
}

check for

updates

Citation: Lee, H.-J.; Shim, J.-S.; Moon, H.-S.; Kim, J.-E. Alteration of the Occlusal Vertical Dimension for Prosthetic Restoration Using a Target Tracking System. Appl. Sci. 2021, 11, 6196. https://doi.org/10.3390/ app11136196

Academic Editors: José João Mendes, Ricardo Castro Alves, Ana Cristina Mano Azul and Alessandra Lucchese

Received: 27 April 2021

Accepted: 30 June 2021

Published: 4 July 2021

Publisher's Note: MDPI stays neutral with regard to jurisdictional claims in published maps and institutional affiliations.

Copyright: (c) 2021 by the authors. Licensee MDPI, Basel, Switzerland. This article is an open access article distributed under the terms and conditions of the Creative Commons Attribution (CC BY) license (https:// creativecommons.org/licenses/by/ $4.0 /)$.

\begin{abstract}
Clinicians and researchers have used various methods to reproduce the maxillomandibular relationship and mandibular movement of individual patients using an articulator, with efforts being made to reduce errors associated with the conventional technique. When a change to a vertical dimension is required during the conventional prosthesis construction process, the maxillary and mandibular casts are mounted on the mechanical articulator using a facebow and bite registration and the elevation of the anterior guide pin of the articulator is used. However, this can inevitably cause errors due to differences between the articulator hinge movement and the actual trajectory of the patient. There has recently been increasing interest in tracking the trajectory of jaw motion of a patient, and this paper presents a new technique for altering the vertical dimension based on the measured trajectory. Target materials for performing tracking are attached to the maxillary and mandibular anterior teeth to record opening and closing movements of the patient's mouth in real time and align the patient's scanned intraoral data or cast data. The movements of the targets are replaced with the movement of the patient's oral scan data. Additionally, then the occlusal vertical dimension is set to a new position based on the obtained trajectory. After determining the optimal vertical dimension with consideration of the space required for restoration, maxillary and mandibular STL files are exported and the designed cast is created using a 3D printer. The printed cast is mounted on an articulator for subsequent procedures. This approach maintains the patient's actual maxillomandibular relationship at various vertical heights and can also reduce the chair time required when adjusting for errors.
\end{abstract}

Keywords: target tracking; digital dentistry; CAD-CAM; occlusal vertical dimension; maxillomandibular relationship

\section{Introduction}

The loss of posterior teeth loss results in the occlusal plane collapsing due to the extraction of the antagonist teeth and the lack of posterior support results in severe wearing of the remaining teeth. When there is insufficient space for a prosthesis due to excessive tooth wear, prosthetic restoration is necessary at the position where the original vertical dimension needs to be restored. The interventions in such cases require consideration of various of factors, including accurate assessment and diagnosis of the vertical dimension, the status of the remaining teeth, and the history of temporomandibular joint disease [1,2]. Determination of vertical dimension is important for fabrication of all restorations. Many techniques have been used for the measurement of the vertical dimension of occlusion such as facial landmarks, swallowing, phonetics, oscilloscope, freeway space, and cephalometric radiographs. There has been much debate in the dental literature concerning whether or not it is permissible to alter the occlusal vertical dimension (OVD). Physiologic OVD can better be described as a range instead of a fixed point or position for most subjects and the width of that comfort zone may vary among individuals according to their adaptive capacity [3]. From the clinical perspective, it is advantageous to consider altering 
the vertical dimension for restorative material, enhancing the esthetic tooth display and allowing for re-establishment of physiologic occlusion [4]. When the orthodontic force is applied to the teeth, periodontal ligament and alveolar bone change occurs abruptly due to the biochemical adaptive response [5,6]. Therefore, it is important to alter the vertical dimension as little as necessary after obtaining an accurate maxillomandibular relationship so that the residual teeth are not affected. Additionally, it is of paramount to mount the study casts in centric relation.

In the traditional method, an impression acquisition process is used to create a plaster cast that reproduces the patient's oral condition, which records the maxillary position based on the patient's cranial base. A facebow and the check-bite in centric relation position are used to transfer the cast to the articulator. A temporary dental prosthesis is then fabricated based on the newly set vertical dimension by raising the incisal guide pin of the articulator [4]. However, for convenience, facebows are not based on the patient's true hinge axis points. In addition, since the patient may have a centric relation-maximum intercuspation (CR-MI) discrepancy, the difference in the positions of the maxilla and mandible may occur when the gypsum model is mounted on the articulator. Therefore, the position and the maxilla-to-mandible vertical dimension of the cast may differ from the patient's actual dimensions [7], and errors may accumulate in the prosthesis being constructed based on this position.

Recent technological advances in digital dentistry have led to various methods for tracking jaw movement being proposed. A target-based method for tracking the movement of a specific reference point using an optical scanner is simpler than other tracking methods that involve mounting bulky and inconvenient equipment on the head, and has the additional advantage of recording the natural movements of patients [8-10].

This article proposes a process for transferring the gypsum cast or three-dimensional (3D) printed cast to the articulator that includes alteration of the vertical dimension. This alteration is determined using a target tracking method that can record the actual jaw motion in patients exhibiting a reduced vertical dimension due to the loss of posterior tooth support and overall wearing of the remaining teeth.

\section{Materials and Methods}

\subsection{Attaching Target Materials to the Anterior Tooth and Tracking Jaw Movements}

Before performing the target tracking process, a patient's remaining teeth, the presence of temporomandibular joint(TMJ) disease, the muscular system, and the current occlusal vertical dimension were evaluated. In the present example, a patient with extreme worn dentition due to the loss of posterior tooth support needed treatment for rehabilitation and did not have any TMJ disease selected. Four 3-mm-diameter non-reflective double circle targets (Target Sticker; Medit Corporation, Seoul, Korea) were attached to the maxillary and mandibular anterior teeth of the patient (Figure 1). Targets were attached after retracting the patient's cheeks using a mouth retractor and drying teeth surface sufficiently. The temporary filling material (Quicks Blue; Denkist, Gyeonggi, Korea) was additionally applied to enhance the adhesive force of the targets.

The anterior dentition is scanned using an intraoral scanner (i500; Medit Corporation, Seoul, Korea) to record the relationship between the targets and the teeth. Casts of the maxillary and mandibular arches using alginate impressions are each scanned and in the maximum intercuspal relationship using a 3D tabletop scanner (Identica Blue; Medit Corporation, Seoul, Korea). An optical scanner (Rexcan CS2; Medit Corporation, Seoul, Korea) is then positioned with its focal length at the patient's anterior dentition to detect the positions of the targets for tracking. The trajectories of the target movements are then tracked continuously with the optical scanner while the patient opens and closes their mouth repeated. After scanning the patient's mouth and cast of the full arch and completing the alignment using the scanned data of the target, the movements of the targets are replaced with the movement of the patient's oral scan data (Figure 2). 

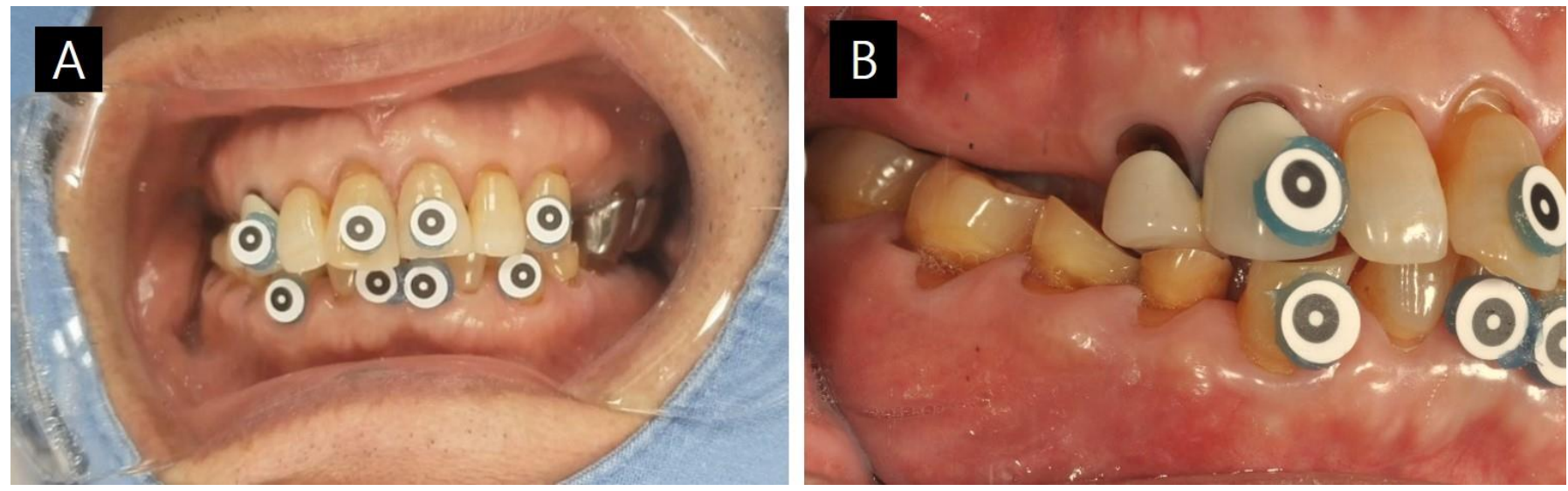

Figure 1. (A) Target tracking materials are attached to the labial surface of the upper and lower anterior teeth. (B) The restoration space is insufficient on the lateral side after losing the antagonist teeth.

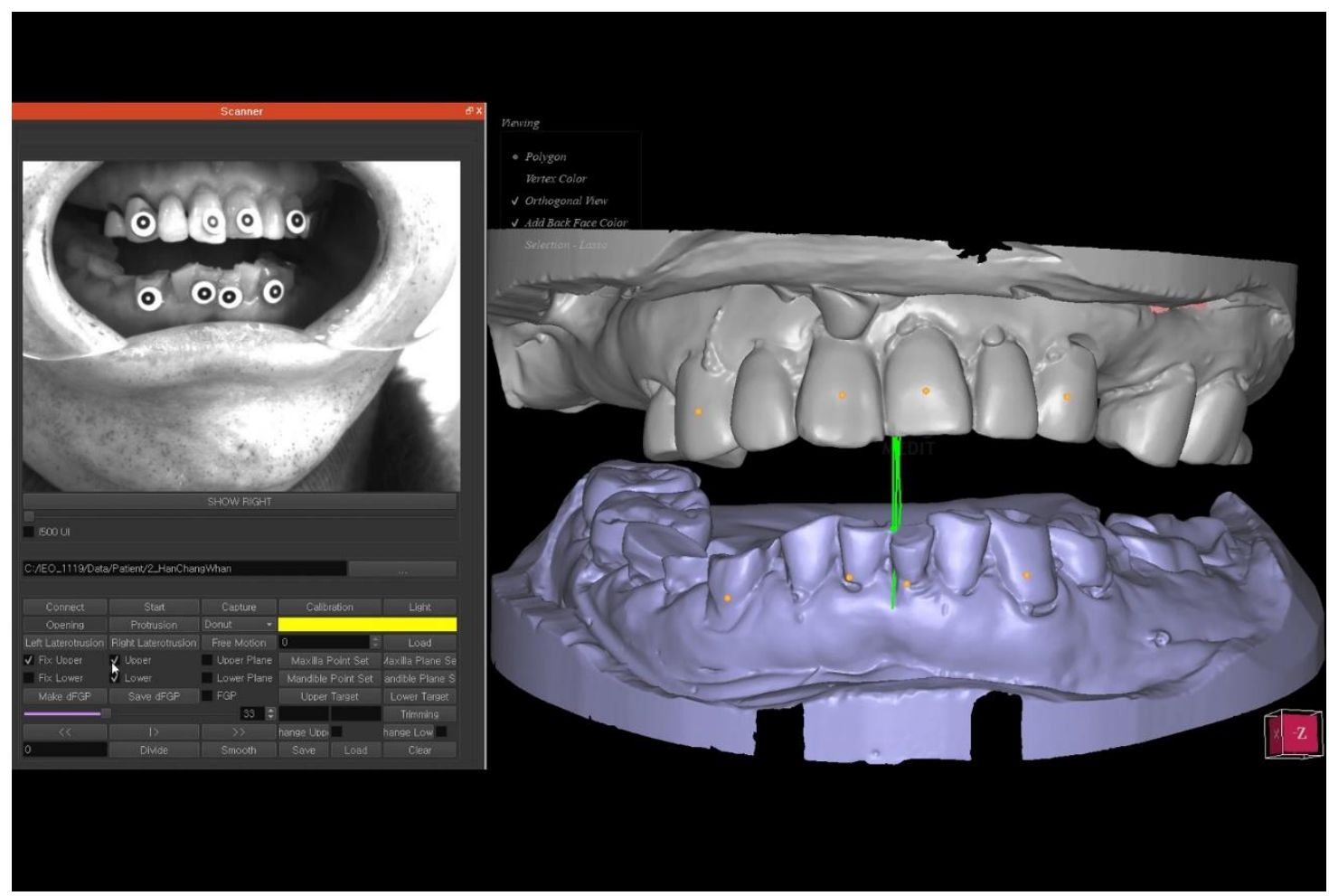

Figure 2. The mandibular movement is recorded in real time and replaced with data of the entire arch model.

The procedure of oral scanning after attaching the targets, target tracking of the anterior teeth using an optical scanner, and replacement of the entire arch data were performed in this case by aligning with referenced target stickers and the same position in the cast [11].

\subsection{Confirmation of $C R-M I$ Discrepancy}

When the patient closes his mouth to the tooth contact position, it is possible to check whether a sliding motion occurs at the first contact position and the presence of CR-MI discrepancy can be checked (Figure 3A). If such a discrepancy is present, a horizontal trajectory (between two green dots) appears and its size can be measured by tracking software (Ezscan8; Medit Corporation, Seoul, Korea). Additionally, then it can be exported by specifying the positions of the maxilla and mandible in the centric relation position (Figure $3 \mathrm{~B}$ ). The centric relation recording followed the chin-point guidance method among 
the traditional methods and the target tracking method was used instead of the bite registration material in the step of recording the relationship between the maxilla and the mandible at the $C R$ position.
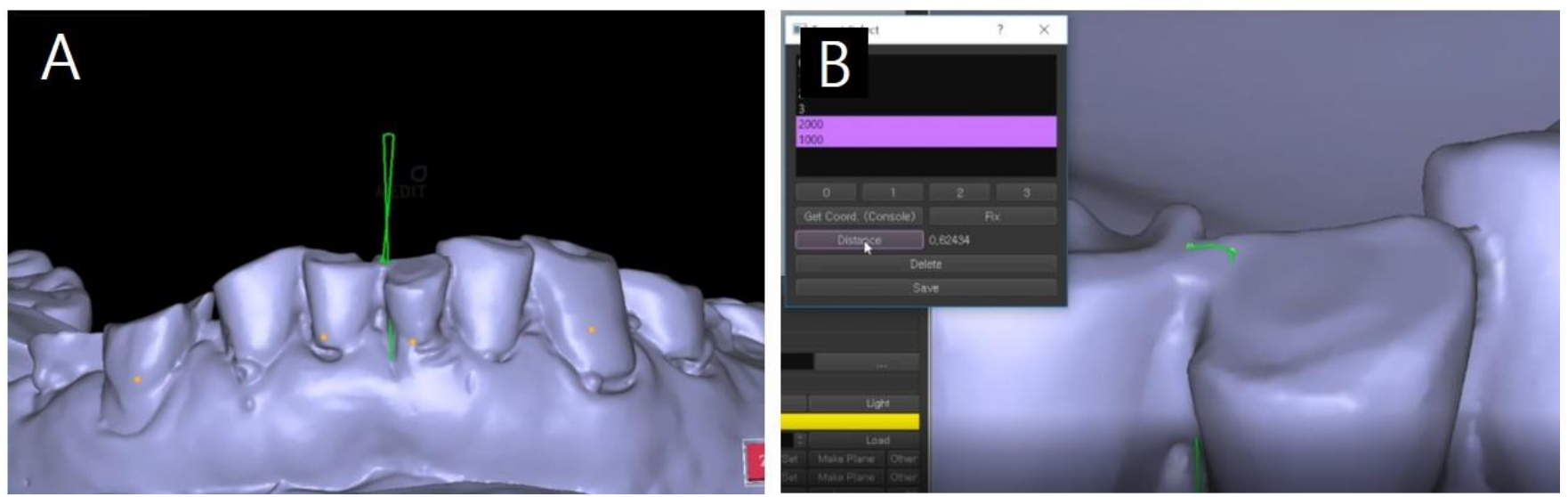

Figure 3. (A) Closing the mouth so that the teeth are in contact can reveal lateral sliding and the presence of a CR-MI discrepancy. (B) Prototype software confirmed that the difference was about $0.6 \mathrm{~mm}$ in this case.

\subsection{Vertical Dimension Alteration}

When adjustment of the occlusal vertical dimension is necessary during prosthetic restoration, the STL file produced based on the opening and closing movements of the patient's mouth can be exported after determining the optimal vertical dimension from consideration of the space required for restoration (Figure 4). Considering the patient's facial height and freeway space, alteration should be the minimum necessary to harmonize dento-facial esthetics and improve the occlusal relationship. Clinicians can select the optimal vertical dimension on a point of the trajectories of jaw motion. Maxillary and mandibular STL files were imported into 3D CAD software (Exocad dentalCAD; Exocad $\mathrm{GmbH}$, Darmstadt, Germany) to reproduce the positional relationship in the digital model with the altered vertical dimension (Figure 5).

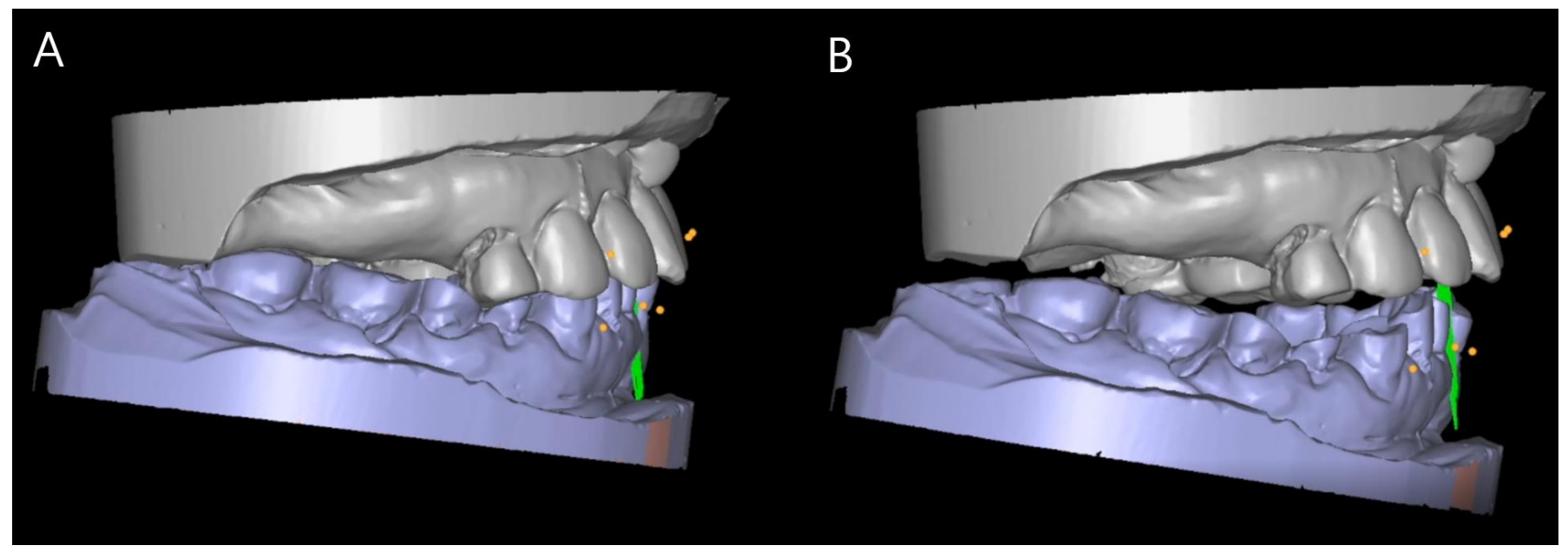

Figure 4. (A) Relationship between maxillary and mandibular arches in the state of maximum intercuspation. (B) An image showing the attempted alteration of the occlusal vertical dimension using digital software. 


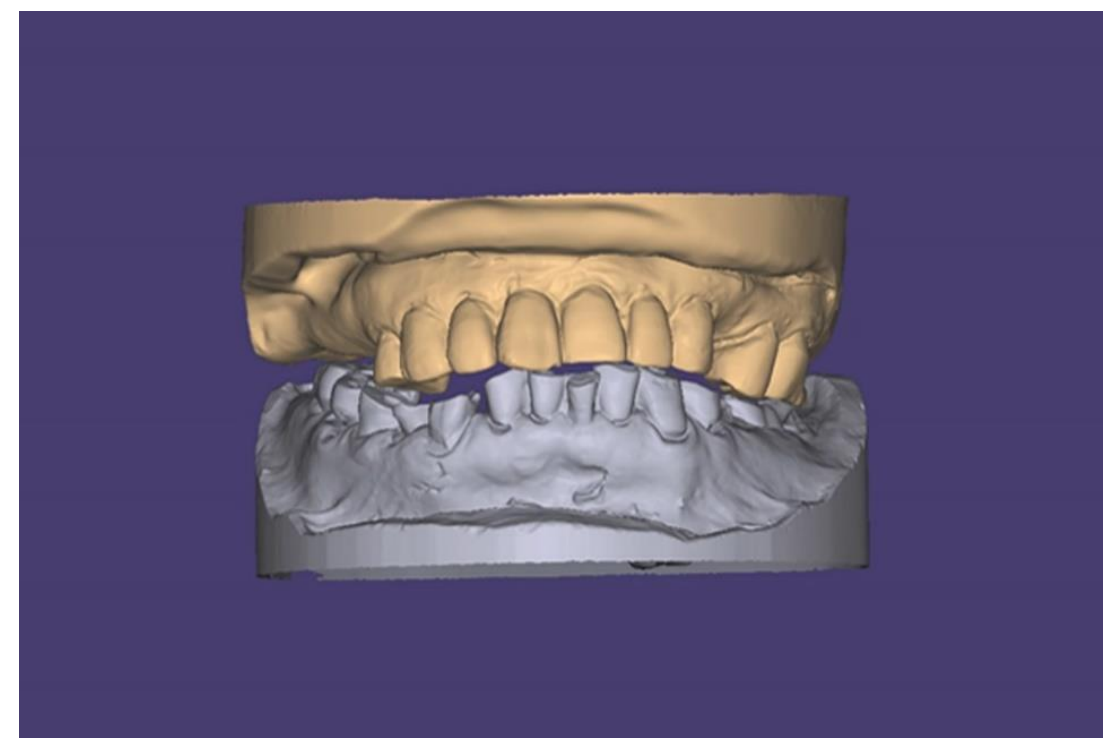

Figure 5. Images loaded from CAD software after exporting data and the relationship between the two arches with the desired occlusal vertical dimension.

\subsection{Fabrication and Mounting of a $3 D$ Printed Cast}

A 3D printed cast was created using the obtained data. In order to physically relocate the position of the cast with the altered vertical dimension, a cast base and mounting pin should be designed using software (Model Creator; Exocad GmbH, Darmstadt, Germany) (Figure 6A). The designed cast was produced using a 3D printer (Form2; Formlabs, Somerville, MA, USA) and 3D printing resin (Grey Resin; Formlabs, Somerville, MA, USA) (Figure 6B).
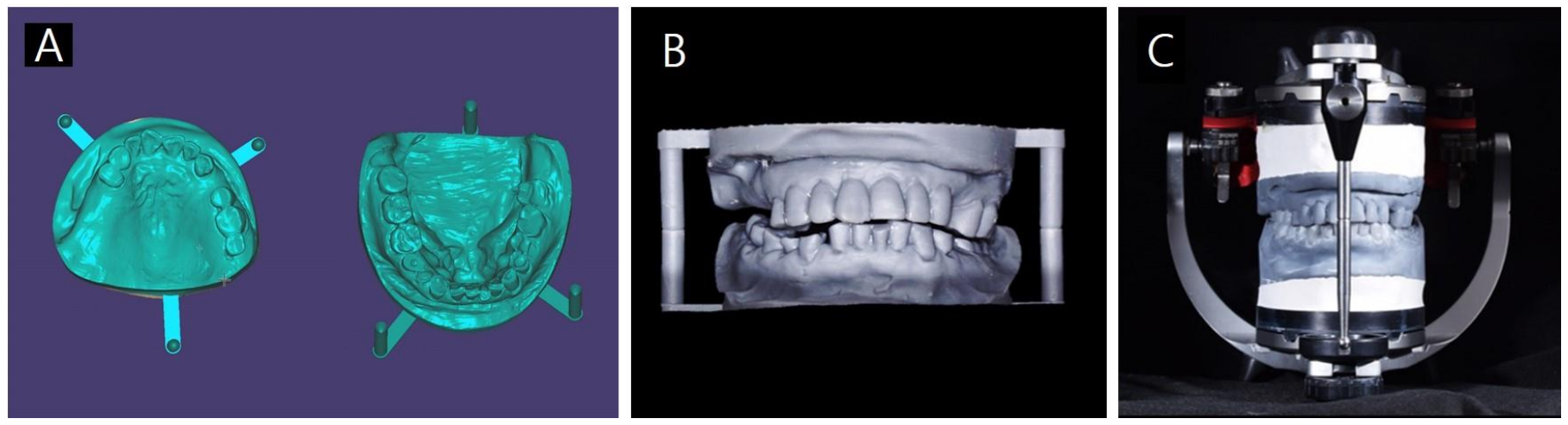

Figure 6. (A) A 3D printed model constructed using the exported data. Three mounting pins were designed to maintain the altered vertical dimension. (B) The 3D printed cast with altered vertical dimension. (C) The 3D printed cast is mounted on the mechanical articulator.

The printed cast with the optimal vertical dimension was mounted on a mechanical articulator while taking into account the positional relationships of the mounting pins (Figure 6C). Diagnostic wax modeling can be put on the cast or the case can be virtually waxed-up before printing the cast and the required prosthesis was then constructed.

\subsection{Mounting Process of the Existing Plaster Cast}

If the existing gypsum cast mounting is required for the laboratory process, a jig for mounting the gypsum cast can be fabricated using the data associated with the optimal vertical dimension (Figure 7A). After designing the jig using the bite splint module of the CAD software (Exocad dentalCAD; Exocad GmbH, Darmstadt, Germany), the jig was 
printed using the 3D printer (Form2; Formlabs, Somerville, MA, USA) and flexible 3D printing resin (Flexible Resin; Formlabs, Somerville, MA, USA) (Figure 7B).
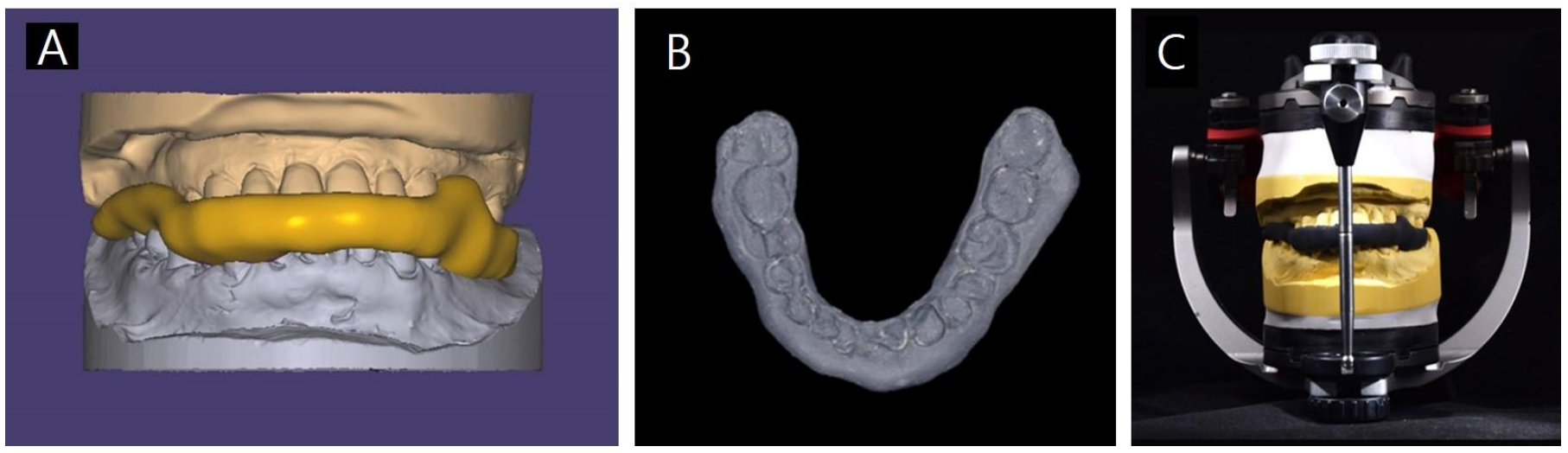

Figure 7. (A) Designing a flexible bite jig consistent with the altered vertical dimension for mounting the plaster cast. (B) The 3D printed bite jig for the gypsum cast mounting process constructed from flexible resin. (C) Mounting the plaster model on the mechanical articulator using the flexible bite jig.

The plaster cast was mounted on the articulator using the printed jig (Figure 7C). Wax was added to the cast for a diagnostic purpose and the required prosthesis was constructed.

\subsection{Preliminary Study for Evaluating Tracking Accuracy}

Preliminary study data for evaluating tracking accuracy according to the arrangement of targets were presented. The static model mounted on the articulator was recorded using the optical scanner while closed position and $4 \mathrm{~mm}$ (distance of tip of upper and lower central incisors) opened position. Targets were attached to the maxillary and mandibular central incisors and canines. The accuracy of target tracking was evaluated at maxillary and mandibular anteriors and the mesio-buccal cusp of the 2nd molars (Figure 8). Tracking data for $7 \mathrm{~s}$ at 50 frames per second was acquired and a total of 350 frames of data were recorded and compared with reference coordinates.

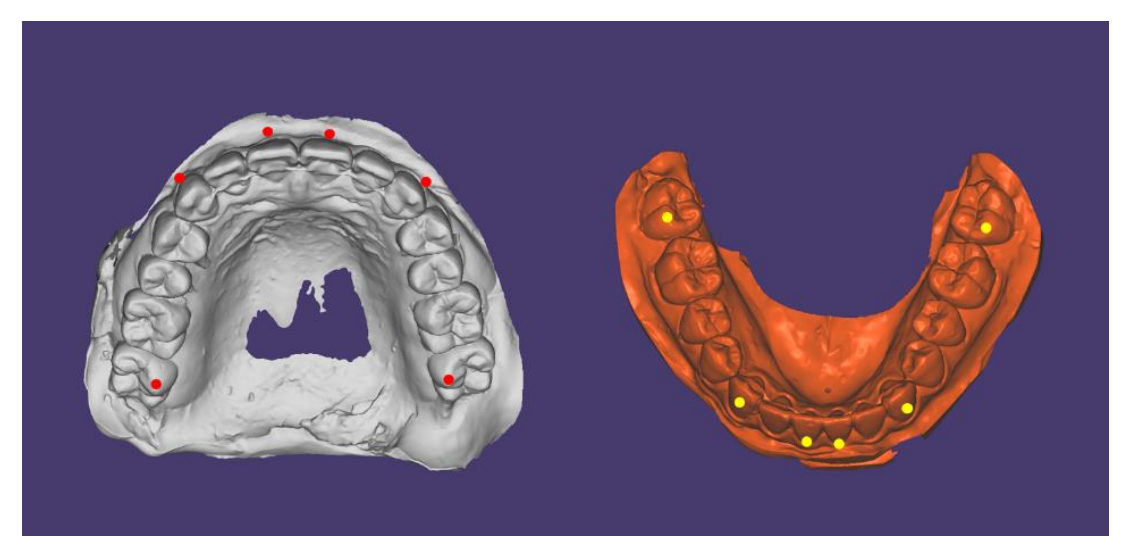

Figure 8. The accuracy of target tracking was evaluated at maxillary and mandibular anterior targets and the mesio-buccal cusp of the 2nd molars.

\section{Results}

The jaw movements of a patient were successfully tracked. Even a small CR-MI discrepancy within $1 \mathrm{~mm}$ could be detected and the optimal occlusal vertical dimension could be determined based on the opening and closing movements of the patient's mouth A 3D printed model or an existing plaster model consistent with the newly set occlusal 
vertical dimension within jaw motion trajectories could be used to mount the model on the articulator and subsequent procedures.

The mean deviation at the closing state was smaller than that of the $4 \mathrm{~mm}$ opening state. The mean deviation of maxillary and mandibular anterior points were smaller than that of the maxillary and mandibular 2nd molar. The accuracy of the tracking data was within $10 \mu \mathrm{m}$ in the anterior target region and within $30 \mu \mathrm{m}$ in the cusp of the second molar, confirming that it showed very high accuracy in all environments (Figure 9).

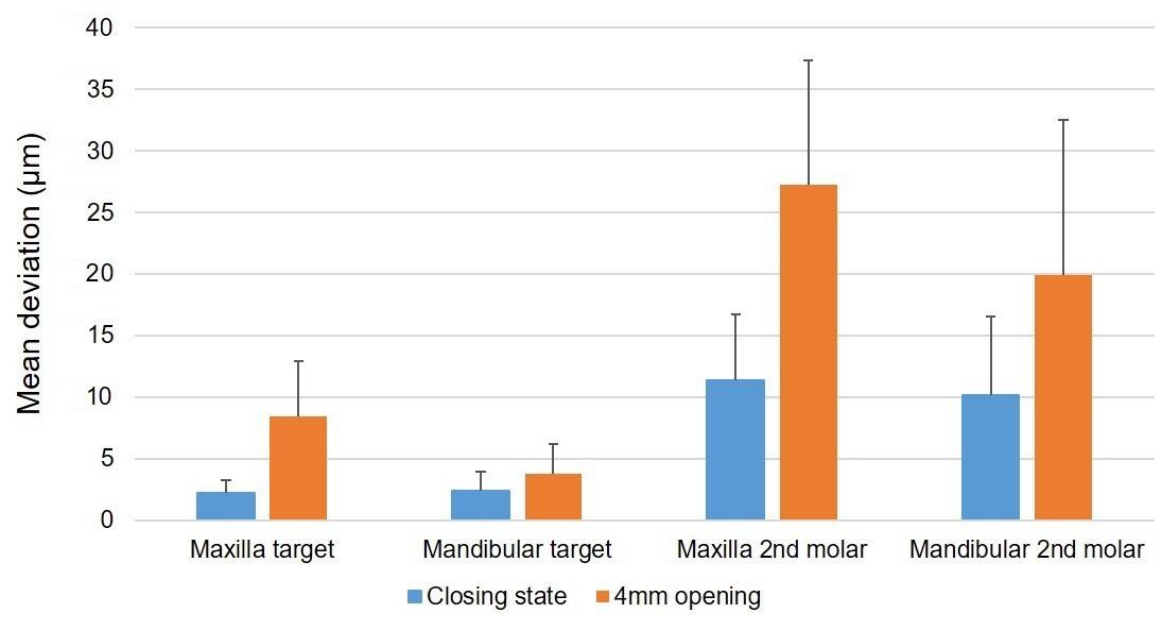

Figure 9. Mean deviation at the closing state was smaller than that of the $4 \mathrm{~mm}$ opening state (mean + standard deviation).

\section{Discussion}

The new technique presented here was able to reproduce the actual opening and closing movements of the patient's mouth, since the positions of the targets attached to the teeth were tracked using an optical scanner. A method was also proposed for confirming the CR-MI discrepancy and transferring the altered vertical dimension to the mechanical articulator. This can be used to fabricate the patient's prosthesis based on the observed trajectory, which allowed the actual maxillomandibular relationship to be maintained despite changes in the vertical dimension even when the patient had a CR-MI discrepancy. In the conventional technique, the facebow and semiadjustable articulator were manufactured based on the assumption that the patient's face and mandibular movements are symmetrical, and so errors can occur when the patient exhibits asymmetry. The occurrence of horizontal displacement during opening and closing trajectories is difficult to reproduce on the articulator. Our novel technique can overcome these limitations of the traditional method since it reproduces the detailed mandibular movements of the patient using digital software.

Another disadvantage of the conventional method is that converting between the digital cast and the physical cast is difficult on the digital articulator embedded in the CAD software. However, in the proposed technique, a 3D printed jig can be used to mount the patient's actual plaster cast in addition to the 3D printed cast. This approach can be very useful in cases that require an existing gypsum cast for the production of different kinds of prosthesis, such as a removable dental or implant prosthesis.

However, our technique also has some limitations. While it is only necessary to attach targets on the anterior teeth for target tracking, which is performed using an optical scanner, the retractor required to keep the targets exposed can interfere with the patient's natural jaw movement. It is important that the targets are in the same position without falling out even during jaw movement. If the position of the targets changes while tracking jaw motion, errors are generated and clinicians have to start over again. There are also limitations associated with the need to have access to optical scanner equipment. In order to fit exactly into the existing plaster casts without any damages, the bite jig needs to be 
flexible. However, there is a possibility that errors may occur due to the flexibility of the bite jig at the same time. It is necessary to confirm that the bite jig should be in the correct position when mounting the casts. Additionally, when flexible materials become too thick, occlusal errors may occur due to the elasticity of the material.

Future studies should assess the accuracy and reproducibility of our new method for altering the vertical dimension and the associated mounting procedure, and consider its compatibility with existing CAD software. The results obtained could lead to this technique having wide clinical applications.

\section{Conclusions}

The present dental technique records the actual mandibular movement of the patient in real time using targets attached to the maxillary and mandibular anterior teeth in patients with reduced vertical occlusion resulting from the loss of posterior teeth and wearing of the remaining teeth. The $3 \mathrm{D}$ printed maxillary and mandible casts can be mounted or a flexible bite jig can be constructed with increased vertical dimension to enable mounting of the existing gypsum cast.

Author Contributions: Conceptualization, H.-J.L. and J.-E.K.; methodology, J.-E.K.; software, H.-J.L. and J.-E.K.; validation, H.-J.L., H.-S.M. and J.-S.S.; formal analysis, J.-E.K.; investigation, H.-J.L.; resources, J.-S.S.; writing —original draft preparation, H.-J.L. and J.-E.K.; writing—review and editing, H.-J.L. and J.-E.K.; visualization, H.-J.L. and H.-S.M.; supervision, J.-E.K.; project administration, J.-E.K. All authors have read and agreed to the published version of the manuscript.

Funding: This research was supported by Basic Science Research Program through the National Research Foundation of Korea (NRF) funded by the Ministry of Education (NRF- 2019R1I1A1A01062792).

Institutional Review Board Statement: Not applicable.

Informed Consent Statement: Not applicable.

Data Availability Statement: The data presented in this study are available on request from the corresponding author.

Acknowledgments: The authors thank Soobok Lee and Sungbin Im from Medit Corporation for providing technical support.

Conflicts of Interest: The authors declare that they have no conflict of interest.

\section{References}

1. Verrett, R.G. Analyzing the etiology of an extremely worn dentition. J. Prosthodont. 2001, 10, 224-233. [CrossRef] [PubMed]

2. Turner, K.A.; Missirlian, D.M. Restoration of the extremely worn dentition. J. Prosthet. Dent. 1984, 52, 467-474. [CrossRef]

3. Rivera-Morales, W.C.; Mohl, N.D. Relationship of occlusal vertical dimension to the health of the masticatory system. J. Prosthet. Dent. 1991, 65, 547-553. [CrossRef]

4. Abduo, J. Safety of increasing vertical dimension of occlusion: A systematic review. Quintessence Int. 2012, 43, 369-380. [PubMed]

5. Matarese, G.; Isola, G.; Ramaglia, L.; Dalessandri, D.; Lucchese, A.; Alibrandi, A.; Fabiano, F.; Cordasco, G. Periodontal biotype: Characteristic, prevalence and dimensions related to dental malocclusion. Minerva Stomatol. 2016, 65, 231-238. [PubMed]

6. Rodriguez y Baena, R.; Pastorino, R.; Gherlone, E.F.; Perillo, L.; Lupi, S.M.; Lucchese, A. Histomorphometric Evaluation of two Different Bone Substitutes in Sinus Augumentation Procedures: A Randomized Controlled Trial in Humans. Int. J. Oral. Maxillofac. Implants 2017, 32, 188-194. [CrossRef] [PubMed]

7. Walker, P.M. Discrepancies between arbitrary and true hinge axes. J. Prosthet. Dent. 1980, 43, 279-285. [CrossRef]

8. Pinheiro, A.P.; Pereira, A.A.; Andrade, A.O.; Bellomo, D. Measurement of jaw motion: The proposal of a simple and accurate method. J. Med. Eng. Technol. 2011, 35, 125-133. [CrossRef] [PubMed]

9. Zafar, H.; Eriksson, P.O.; Nordh, E.; Häggman-Henrikson, B. Wireless optoelectronic recordings of mandibular and associated head-neck movements in man: A methodological study. J. Oral. Rehabil. 2000, 27, 227-238. [CrossRef] [PubMed]

10. Kim, J.E.; Park, J.H.; Moon, H.S.; Shim, J.S. Complete assessment of occlusal dynamics and establishment of a digital workflow by using target tracking with a three-dimensional facial scanner. J. Prosthodont. Res. 2019, 63, 120-124. [CrossRef] [PubMed]

11. Han, C.G.; Park, Y.B.; Shim, J.S.; Kim, J.E. Restorative Space Analysis by Jaw Motion Tracking Using a Template in Completely Edentulous Patients. Appl. Sci. 2021, 11, 3933. [CrossRef] 